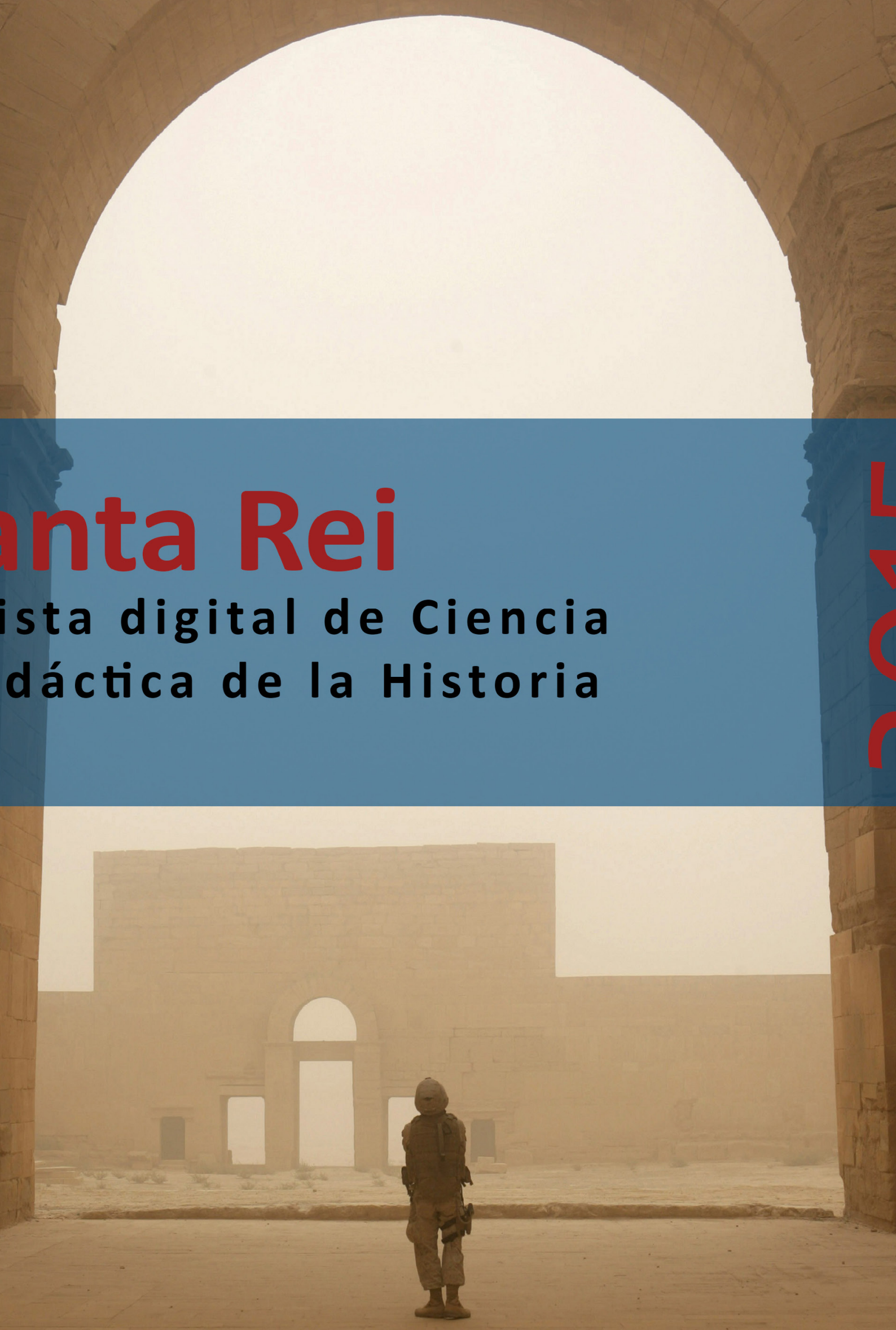

Revista digital de Ciencia y Didáctica de la Historia 


\section{Panta Rei \\ Revista Digital de Ciencia \\ y Didáctica de la Historia}

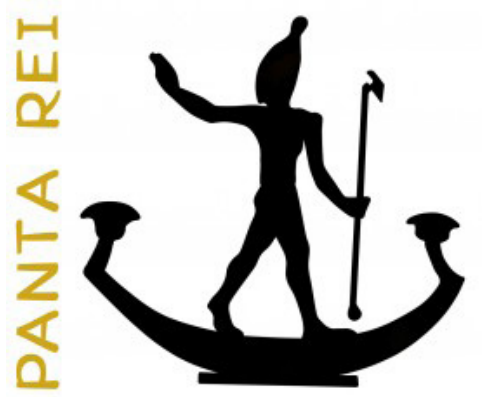

\section{5}

Revista anual

Fecha de inicio: 1995

Revista Panta Rei. pantarei@um.es

Edita:

Centro de Estudios del Próximo Oriente y la Antigüedad Tardía - CEPOAT

Edificio Universitario Saavedra Fajardo.

Universidad de Murcia

C/ Actor Isidoro Máiquez, 9

30007 - MURCIA - ESPAÑA

Teléfono: (+34) 868883890

cepoat@um.es

Web: www.um.es/cepoat/pantarei

Edición 2015

ISSNe: 2386-8864

ISSN: 1136-2464

Depósito legal: MU-966-1995
cepoAt

UNIVERSIDAD DE MURCIA centro de estudios del próximo oriente y la antigüedad tardía

En Portada: Ruinas de Hatra (Irak) (Fotografía de: Lance Cpl. Albert F. Hunt, U. S. Marine Corps).

Responsables de los textos: Sus autores.

Responsable de la presente edición: Consejo Editorial Panta Rei. 


\section{CONSEJO DE REDACCIÓN}

\section{Coordinador editorial}

Egea Vivancos, Alejandro

[Didáctica de las Ciencias Sociales, UMU]

\section{Editores}

Botí Hernández, Juan Jesús

[CEPOAT, UMU]

Meseguer Gil, Antonio José

[CEPOAT, UMU]

Sáez Giménez, David Omar

[CEPOAT, UMU]

Sánchez Mondéjar, Celso Miguel

[CEPOAT, UMU]

\section{Secretaria}

Arias Ferrer, Laura

[Didáctica de las Ciencias Sociales, UMU]

\section{Responsable informático}

Martínez García, José Javier

[CEPOAT, UMU]

\section{Traducción y corrección lingüística}

Martínez Martínez, Cristina

[Sociedad Española de Lenguas Modernas]

\section{CONSEJO ASESOR}

Albero Muñoz, M. ${ }^{a}$ del Mar [H. ${ }^{a}$ del Arte, UMU]

Chapman, Arthur

[History Education, UCL, Reino Unido]

Cobacho López, Ángel

[Derecho, UMU]

Egea Bruno, Pedro M. ${ }^{\text {a }}$

[Historia Contemporánea, UMU]

García Atienzar, Gabriel

[Prehistoria, UA]

González Monfort, Neus

[Didáctica de las Ciencias Sociales, UAB]

Haber Uriarte, María

[Prehistoria, UMU]

Irigoyen López, Antonio

[Historia Moderna, UMU]

Mahony, Simon

[Digital Humanities, UCL, Reino Unido]

Marsilla de Pascual, Francisco Reyes

[Técnicas historiográficas, UMU]

Miralles Maldonado, José Carlos

[Filología Clásica, UMU]

Molina Gómez, José Antonio

[Historia Antigua, UMU]

Noguera Celdrán, José Miguel

[Arqueología, UMU]

Pérez Molina, Miguel Emilio

[Filología Clásica, UMU]

Prados Martínez, Fernando

[Arqueología, UA]

Sánchez Ibáñez, Raquel

[Didáctica de las Ciencias Sociales, UMU]

Sancho Gómez, Miguel Pablo

[Educación, UCAM]

Vilar García, María José

[Historia Contemporánea, UMU] 

Artículos

El patrimonio arqueológico iraquí y su destrucción a lo largo del tiempo.

Fernando Espejel Arroyo.

Tacita Muta y el silencio femenino como arma del patriarcado romano.

Sara Casamayor Mancisidor.

Espartaco en la arqueología.

Juan Luis Posadas.

La batalla de Andagoste.

Luis Amela Valverde.

Las trabas para una formación arqueológica inclusiva.

Ana Samaniego Espinosa.

Repensando las narrativas nacionales: Un análisis del origen, transmisión e influencia en el aprendizaje histórico.

César López Rodríguez

El profesor como predicador, provocador y ermitaño. Un pre-texto para pensar las tareas de la didáctica de la historia.

Enrique Ibáñez Rojo

Entrevista

Evaluando el desarrollo de la competencia histórica en la enseñanza de la Historia en España. Entrevista a Jesús Domínguez Castillo.

María José Morales Rodríguez.

Reseñas

II Congreso de Jóvenes Investigadores del Mundo Antiguo de la Universidad de Murcia.

Pedro David Conesa Navarro y Juana M. ${ }^{a}$ Martínez Molina.

II Jornadas Internacionales de Didáctica de la Historia.

Eusebio Manuel Vidal Abellán.

Dölger, F. J. (2015). La luz de Cristo. Madrid: Ediciones Encuentro. 98 págs.

Alejandro Ruiz Sánchez.

Cooper, H. (ed.) (2013). Teaching History Creatively. Londres: Routledge. 185 págs.

Marta Gil de Sola Bellas.

El arte paleolítico en El Carnicero de Chabrol.

Alberto Lombo Montañés.

Normas de publicación/Publishing rules 



\title{
Evaluando el desarrollo de la competencia histórica en la enseñanza de la Historia en España Entrevista a Jesús Domínguez Castillo
}

\author{
María José Morales Rodríguez \\ Universidad de Murcia
}

\begin{abstract}
Para citar esta entrevista: Morales Rodríguez, M. ${ }^{a}$ J. (2015, noviembre). Evaluando el desarrollo de la competencia histórica en la enseñanza de la Historia en España: Entrevista a Jesús Domínguez Castillo. Panta Rei. Revista Digital de Ciencia y Didáctica de la Historia, 111120.

ISSNe: 2386-8864

DOI: http://dx.doi.org/10.6018/pantarei/2015/8
\end{abstract}

Jesús Domínguez Castillo, nacido en Arnedo (La Rioja) en 1952, representa desde hace varias décadas una figura destacable en el ámbito de la enseñanza de la Historia en nuestro país. Sus aportaciones a nivel docente e investigador y sus múltiples colaboraciones en proyectos de la más diversa índole, le han llevado a ser uno de los mayores expertos en cuanto al desarrollo y la evaluación de las competencias involucradas en el proceso de pensar históricamente. Licenciado en Filosofía y Letras, sección Historia, en 1974 por la Universidad de Zaragoza, amplió su formación en 1984 con un Máster en Educación de la Historia en el University of London, Institute of Education. Posteriormente, en 1993, obtuvo el grado de Doctor por la Universidad Autónoma de Barcelona, gracias a su trabajo sobre la explicación histórica, con el que exploró la contribución que la enseñanza de los conceptos interpretativos y los procedimientos metodológicos propios de la Historia pueden aportar al proceso de aprendizaje en las etapas obligatorias. Como Catedrático de Geografía e Historia en la Educación Secundaria ha alternado la docencia con responsabilidades en el Ministerio de Educación de España. Por un lado, colaboró como consejero y asesor en el diseño y desarrollo del currículo de la LOGSE, y también fue jefe de redacción de la Revista de Educación. Por otro, durante un periodo de cuatro años (2008/2012) coordinó en España varios estudios de evaluación internacionales, tales como PIRLS (Progress in International Reading Literacy Study) y TIMSS (Trends in International Mathematics and Sciences Study). Pero su labor y entrega a nivel profesional no acaba aquí. Ha sido también profesor de Didáctica de la Historia en cursos de Máster de Educación en instituciones tan prestigiosas como University of London y, más recientemente, en la Universidad Nebrija de Madrid. El currículum de Jesús Domínguez incluye, además, una veintena de artículos y trabajos en libros colectivos sobre Didáctica de la Historia y, en particular, sobre el aprendizaje y la evaluación de la disciplina en los niveles de enseñanza secundaria. Actualmente mantiene una relación de colaboración con la Universidad Nebrija, así como también continúa desempeñando tareas de investigación de la mano del proyecto COMPSOCIALES y de la Red14 de Investigación en Enseñanza de las Ciencias Sociales. En los últimos años ha centrado su trabajo en la evaluación de la Historia como materia de enseñanza, siendo fruto de ello la reciente publicación de su libro Pensamiento histórico y evaluación de competencias (2015). De este modo, la amplia y heterogénea formación de Jesús Domínguez y su dilatada experiencia, tanto en el plano 
nacional como en el internacional, en lo que a la enseñanza de Historia se refiere, posibilita que el autor pueda ser identificado como una persona clave a la hora de evaluar la situación actual del pensamiento histórico en nuestro país.

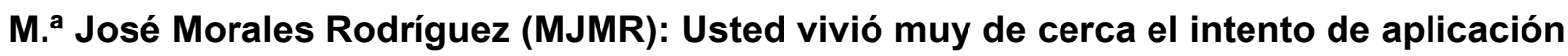
del proyecto Historia 13-16 en nuestro país. Han pasado décadas y los centros educativos prácticamente no se han visto influidos por su espíritu. ¿Nos puede contar brevemente en qué consistió y qué dificultades encontró para su desarrollo?

Jesús Domínguez Castillo (JDC): El método Historia 13-16 procede originalmente de un proyecto británico History 13-16 que se puso en marcha en Reino Unido en 1972, concretamente en Inglaterra, y que comenzó a ser aplicado en las clases en 1974. Este proyecto recibió el nombre de la edad del alumnado al que iba dirigido, estudiantes de 13 a 16 años. Fue un proyecto muy exitoso en la educación británica, tanto que ha llegado a ser trabajado prácticamente por un tercio de los adolescentes. En España, en la década de los setenta, un grupo de profesores de la Universidad de Barcelona conoció el proyecto y le pareció muy interesante. En consecuencia iniciamos la experimentación de una parte de los materiales propios del proyecto inglés: la unidad de introducción a la Historia y una unidad "en desarrollo": La historia del vestido.

Una de las características del proyecto británico es que se centra especialmente en el estudio de la Historia como forma de investigar y de conocer el pasado. Es decir, qué es la Historia, cómo trabajan los historiadores y en qué se fundamenta lo que dicen. Es una forma de trabajo distinta a la que tienen por ejemplo los biólogos, los físicos, los químicos, etc., y es lo que precisamente la caracteriza como forma de adquirir conocimiento sobre nuestro pasado. Hay un ejercicio que consiste en presentar al alumnado un sobre con una serie de materiales, que simulan un caso policial de investigación en el cual se ha encontrado a un chico muerto con una serie de documentos en su macuto: algunos carnés, tiques, hojas de agenda de sus planes, etc., de forma que el alumnado tiene que plantear preguntas para descubrir, a partir de esos documentos, qué pudo ocurrir, quién era ese chico, qué vida llevaba, qué hacía, por qué estaba allí... Ese trabajo de detective después se aplica a la Historia, donde nos encontramos con situaciones similares, como por ejemplo, que se ha hallado el cadáver bastante conservado aún de una persona que vivió hace mil años en unas circunstancias determinadas y con unos restos concretos. De nuevo se le plantean preguntas al alumnado sobre quién era, si era hombre o mujer, cuántos años podía tener, de qué se alimentaba, si el origen de su muerte pudo ser por sacrificio ritual, etc. Ese es un poco el planteamiento inicial, de forma que el curso introductorio de Historia 13-16 era muy atractivo.

Otra de las características importantes del proyecto es que no se basa en una exposición cronológica de toda la Historia, tal y como hasta entonces estábamos habituados. En su lugar, el programa tiene varios tipos de unidades: "Estudios en desarrollo", en los que se selecciona un tema en concreto como puede ser la medicina o la energía -aquí en España se escogió el tema del vestido-, y se estudia cómo ha evolucionado a lo largo del tiempo, haciendo un recorrido de las grandes etapas de la Historia a través de ese tema particular. Ofrece también "Estudios en profundidad" de épocas determinadas, como por ejemplo un estudio sobre la Revolución industrial o un estudio sobre el Oeste americano, una unidad de investigación sobre historia local ("La historia a nuestro alrededor) y varios estudios de historia del mundo contemporáneo, como el conflicto árabe-israelí, o el avance hacia la unidad europea. Cada uno de estos estudios se trabaja durante unos meses, lo que permite trabajar con cierta profundidad los temas. Es, por tanto, una visión de la enseñanza de la Historia mucho más reflexiva, que hace pensar y trabajar con los instrumentos que tiene el historiador, de forma simulada obviamente, pero utilizando materiales iguales o bastante cercanos a los reales.

MJMR: Debido a su formación y experiencias conoce de primera mano el sistema educativo inglés. En relación con la pregunta anterior, ¿cuáles considera que fueron las 
principales diferencias que hicieron que este proyecto no tuviera la misma acogida o el mismo desarrollo en España que en Reino Unido?

JDC: En principio, en la década de los setenta y principios de los ochenta, el Reino Unido no tenía un plan de estudios o un currículo obligatorio, de forma que los centros podían elegir su plan de estudios, sus programaciones didácticas, etc. y la única restricción que tenían era que los alumnos, al final de la secundaria obligatoria, debían pasar un examen si querían tener un certificado que reconociera esta formación. En consecuencia, había distintas ofertas, distintos tribunales de exámenes (Cambridge, universidades del sur de Inglaterra...) y cada uno podía ofrecer incluso hasta dos o tres opciones, de manera que podías establecer un programa de Historia, de acuerdo con un tribunal y preparar a tus alumnos para el examen correspondiente. Así, para el caso de History 1316 se acordó con uno de los tribunales un tipo de examen, en el cual, -y aprovecho ya para hablar de evaluación-, uno de los tres ejercicios era el denominado "ejercicio desconocido". Se llamaba así porque incluía fuentes históricas (textos, fotografía, gráficos, etc.) sobre un tema concreto que el alumnado no había estudiado previamente (por ejemplo el bombardeo nazi de la ciudad de Londres en 1940), de forma que lo que se evaluaba era su capacidad de comprensión lectora, su capacidad de relacionar, de analizar las fuentes, de comparar, de cotejarlas, de interpretarlas en el contexto histórico en el que se insertaban, etc. No eran preguntas de memorizar, no tenían que saber nada de ese tema en concreto, que era muy restringido, aunque desde luego debían conocer los hechos y rasgos principales de la época.

En España este planteamiento no llegó a consolidarse, posiblemente por nuestra tradición enciclopedista de la enseñanza, que nos lleva a tener una sensación de vacío si se nos queda en el tintero un periodo importante o si no se sabe nada de Francia, o de Europa en un periodo determinado. No concebimos la Historia sin hacer un repaso general a todo, a todos los periodos y en todas las dimensiones: la política, la economía, etc. Y esa tradición, es difícil de romper.

Desde que se estableció la Historia como asignatura en el siglo XIX, según han investigado algunos autores como Ramón López Facal (2003), su enseñanza se planteó como forma de insuflar el patriotismo a los futuros ciudadanos dentro del Estado Liberal que se creó en aquellos momentos, inculcando amor a la patria y conocimiento de nuestras identidades y raíces. Lo mismo se puede decir que ocurrió en Inglaterra, sin embargo en nuestro caso, desde esos orígenes hasta nuestros días, creo que la enseñanza de la Historia ha variado muy poco: hay que estudiarlo todo, aunque sea superficialmente o por encima, lo que apenas tiene ninguna utilidad. Hoy en día se habla de competencias, de poder aplicar los conocimientos a la realidad y a las situaciones y problemas que se nos presentan. En este sentido, la Historia que se enseña ofrece poca formación útil para los ciudadanos, para afrontar situaciones diversas, complejas y cambiantes.

MJMR: Desde hace varios años, es notable la influencia que están teniendo los planteamientos didácticos anglosajones en el devenir de las investigaciones sobre enseñanza de la Historia en países como el nuestro. Sin embargo, en las aulas sigue predominando un enfoque teórico, con aprendizajes de base memorística articulados a partir de las directrices asumidas por el libro de texto y, cómo no, evaluados a través del tradicional examen que se mantiene ajeno a los procedimientos, las actitudes y la contextualización social de los contenidos. ¿Cuáles considera que son los principales obstáculos para que en nuestro sistema educativo se implementen planteamientos didácticos, como los que menciona, en los que se prima el desarrollo de la competencia histórica?

JDC: Resulta verdaderamente difícil responder a esta pregunta. Por un lado, cabe destacar que en el año 1990 se creó un currículo bastante flexible y abierto con la Ley Orgánica General del Sistema Educativo, la conocida LOGSE (Ley Orgánica 1/1990), con el que no se determinaba qué contenidos había que dar en cada curso, sino que estos eran presentados de forma global, a fin de que los centros educativos tuvieran mayor autonomía a nivel organizativo y curricular. 
Así, se estableció una etapa común, la Educación Secundaria Obligatoria (ESO), que era en cierta forma igual para todos hasta los 16 años. Esta etapa contaba con un plan de estudios para Ciencias Sociales, Geografía e Historia, que poseía una particularidad importante: establecía pocos contenidos para las programaciones didácticas, dejando atrás el carácter exhaustivo y atomizado que venía siendo habitual en los currículos. Al mismo tiempo, se introdujo "desde arriba", por así decirlo, la necesidad de que en las aulas se enseñaran destrezas, procedimientos, actitudes y valores. Y, a pesar de todo ello, no se consiguió. Por otro lado, el Ministerio de Educación incluso financió grupos de innovación educativa para elaborar materiales que llevaran ese espíritu de la LOGSE a la realidad práctica de las aulas. Desgraciadamente tales materiales se orientaron a un profesorado minoritario y solo algunas editoriales, como Akal por ejemplo, se interesaron por su publicación, quedando reducida esta aspiración del Ministerio a un ámbito muy restringido de docentes. Además de las autoridades educativas, considero por tanto que los docentes y también las editoriales tenemos ahí una responsabilidad importante, y debemos hacer un esfuerzo para buscar fórmulas alternativas que permitan una enseñanza de la historia menos basada en la memorización y mucho más estimulante para los estudiantes. En consecuencia, actualmente el libro de texto continúa siendo como la "Biblia" o el "catecismo" en formato resumen que acompaña al programa oficial que, a su vez, se muestra muy cerrado y establecido.

Y así, estamos ante un círculo vicioso difícil de romper, ya que ni siquiera con la voluntad del Ministerio de Educación de intervenir "desde arriba" para introducir en las aulas una enseñanza más activa y participativa, fuimos capaces de transformar la enseñanza de la Historia. Frente a ello, hay que destacar que en el ámbito británico no se partió "de arriba", elaborando un currículo para, de allí, "bajarlo" a las aulas. De hecho, el Ministerio no participó directamente en el History 13-16, sino que fue un proyecto financiado por una agencia pública de desarrollo del currículo. Esa experiencia magnífica, poco a poco, se fue difundiendo gracias a la realización de cursos y la valoración muy positiva de sus resultados. El sistema en España es muy distinto y por ello es difícil extrapolar este proceso. No obstante, la profunda reflexión que generó ese proyecto británico y los trabajos de Denis Shemilt -director y evaluador del proyecto- desde la Universidad de Leeds, así como los de otros muchos didactas, propiciaron una reflexión teórica muy importante que es precisamente la que nos ha llegado a nosotros y a otros países como Canadá, Estados Unidos, Australia, Holanda, Portugal y Brasil, entre otros.

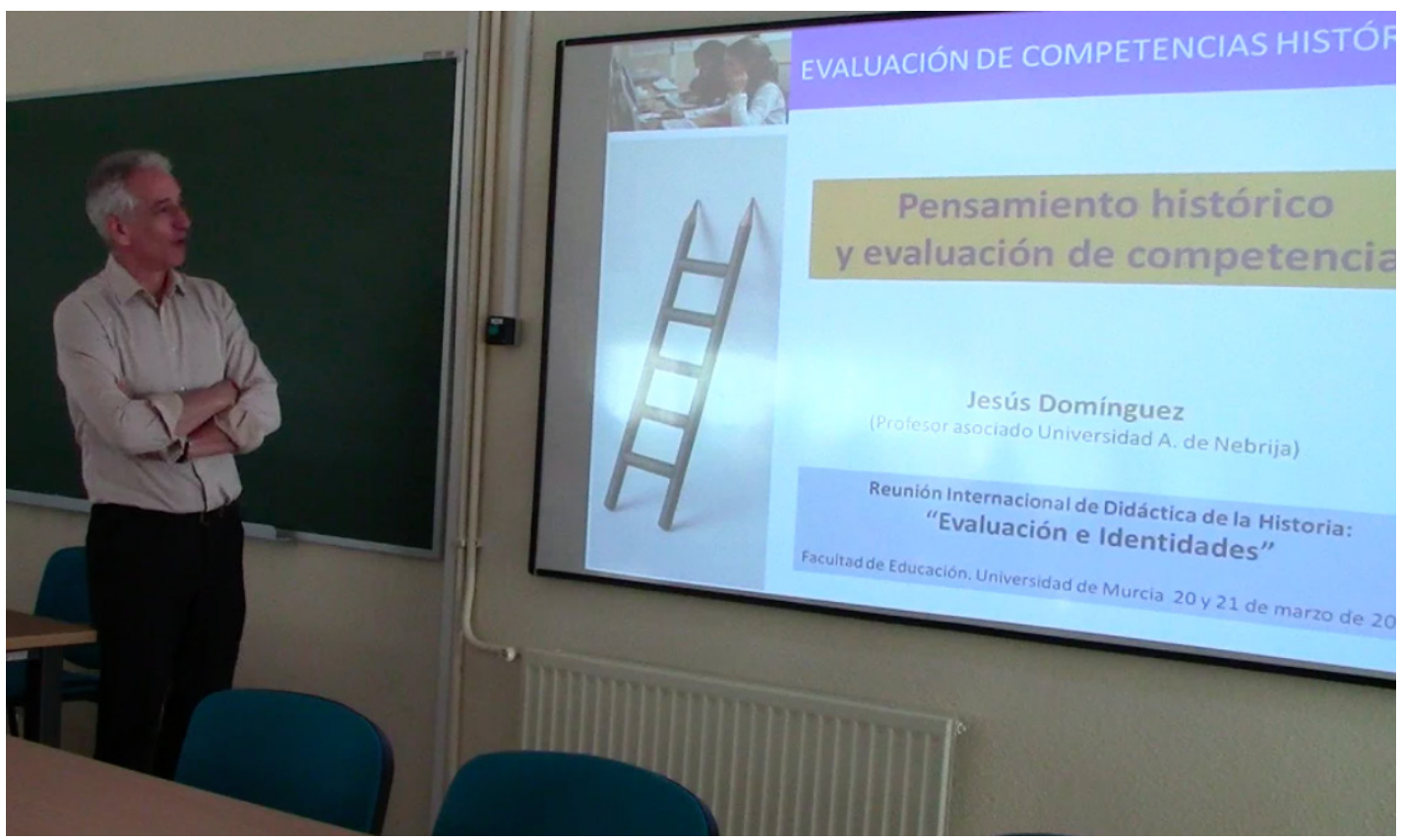

Figura 1: Participación de Jesús Domínguez en la Reunión Científica Internacional de Didáctica de la Historia “Evaluación e identidades". Murcia. 20-21 de marzo de 2014. (Fuente: Alejandro Egea). 
MJMR: Es cierto que en los últimos años está aumentando el volumen de producción científica sobre innovaciones e investigaciones educativas, cuyo objeto de estudio se corresponde con el proceso de enseñanza y aprendizaje de las Ciencias Sociales. Pese a ello, en lo referente a la didáctica y más acusadamente en la etapa de Educación Primaria, la realidad es que en la mayoría de aulas se mantienen como cápsulas atemporales, en las que apenas algunos aspectos innovadores llegan a ponerse en práctica. ¿Cuáles considera que son las principales dificultades que encuentra la producción científica en su camino hacia las aulas?

JDC: Sin duda, el trabajo en el aula exige mucho al profesorado, en el sentido de que te encuentras ante veinticinco o treinta personas que, aunque sean jóvenes o incluso niños, tienen sus exigencias y si lo que les planteas no les "engancha", entonces el proceso de enseñanzaaprendizaje se convierte en una tarea poco fructífera. Considero que ante esa inseguridad que el docente casi siempre suele tener, es muy difícil buscar alternativas a corto plazo. Se necesita mucho esfuerzo y dedicación para ofrecer unos materiales distintos que permitan llevar la innovación a las aulas. Personalmente me ocurre, pues a pesar de que teóricamente estoy muy imbuido de estos planteamientos didácticos, a la hora de plasmarlos en actividades y ejercicios concretos para el aula, se me hace difícil. Por poner un ejemplo, he estado casi dos años trabajando en el libro sobre la evaluación de la Historia, que has mencionado en la presentación, y una buena parte del tiempo la he dedicado a elaborar una prueba que pueda ilustrar en la práctica toda esta reflexión teórica sobre qué y cómo evaluar el conocimiento histórico. Ahora mismo estamos revisando con colegas de la Universidad de Murcia cómo resultó la aplicación piloto de dicha prueba y todavía encontramos problemas en algunas de las preguntas, porque están mal formuladas, porque el alumno las entiende de una manera distinta a como nosotros pensábamos, etc. Eso puede dar una idea de lo difícil que es llevar la reflexión didáctica al aula. Es evidente que si se pregunta al alumno por la fecha en que llegó Cristóbal Colón a América o que identifique invenciones técnicas de la Revolución Industrial, la prueba es relativamente fácil de elaborar y de corregir. En cambio si, a partir de un texto, se pregunta, como hacemos en la prueba, qué hechos y circunstancias fueron causa, pongamos por caso, del aumento de la explotación infantil durante la Revolución Industrial, y qué conexiones e influencias hay entre esas causas, entonces tanto el diseño de la prueba como su corrección se complican bastante. En suma, elaborar una prueba o unos materiales para la enseñanza del pensamiento histórico es un trabajo difícil y duro.

Es imprescindible que en los cursos de Máster para Secundaria, y en el Grado de Primaria, se elaboren materiales y se preparen experiencias didácticas para proceder después a su análisis y evaluación. Diría que, durante la formación docente, el estudio teórico debe estar muy vinculado a la práctica, porque al final es esa práctica en el aula la que enriquece y retroalimenta la innovación y la investigación. Creo que esa es la cuestión principal. Recuerdo que en 1982, cuando hacía un Máster en el Institute of Education de la Universidad de Londres, conocí a compañeros recién graduados en historia, lengua, matemáticas, etc. que estaban haciendo un curso que les habilitaba para impartir clases en secundaria. Fue mi primera noticia de este tipo de cursos. Era muy práctico, y con frecuencia acudían a los centros, para los que debían diseñar actividades didácticas específicas. Frente a ello, el curso de master que yo hacía, era para profesores más experimentados, y tenía un fuerte componente teórico y de investigación. Ya entonces sentí no haber tenido la oportunidad de tener esa formación práctica previa.

MJMR: Quizás ese planteamiento integrador de teoría y práctica es lo que también necesitan los alumnos de los niveles de enseñanza obligatoria para abandonar esa base memorística que ya hemos comentado. Respecto a ello, en una de sus publicaciones (Domínguez, 1989) resalta la necesidad de que el alumnado aprenda a mirar a su alrededor con "ojos históricos". En pro de conseguir esa mirada histórica, ¿considera, desde su experiencia, que para la etapa de Educación Primaria deberían tener mayor peso los 
contenidos sustantivos propios de la disciplina de la Historia o, por el contrario, tendríamos que poner el énfasis en aquellos contenidos metahistóricos de carácter más estratégico?

JDC: Para dar respuesta a esta pregunta recordaría la experiencia de mis hijos en colegios ingleses en los años noventa. Uno de ellos tenía nueve años cuando llegó y cursó los tres últimos años de primaria. En esos años debió tener, en cada curso, unas dos o tres horas semanales de clase de historia, durante un trimestre o poco más. Pues bien, en todo ese tiempo, recuerdo que estudió tres grandes unidades de historia: el ejército romano, los aztecas, y el bombardeo nazi en las ciudades británicas de 1940, o sea, ni siquiera la Segunda Guerra Mundial, solo el bombardeo de la aviación alemana sobre Londres. Todavía hoy, cuando hablamos de esa época, recuerda su experiencia en clase con satisfacción, pues adquirió un conocimiento significativo, aunque limitado, de esas épocas históricas a través de temas muy, muy específicos. No estudió la cronología de la historia universal, ni siquiera, la de los grandes periodos históricos, lo que puede ser sin duda una laguna importante, pero tuvo un primer contacto y aproximación al pasado, significativo para él, que le generó placer por estudiar y comprender a los seres humanos en otras épocas y circunstancias.

Con ese ejemplo, quiero destacar que es necesario reducir la extensión de los programas para poder enseñar cómo es el conocimiento histórico, cómo es, por ejemplo, el trabajo con las fuentes (textos, imágenes y objetos,) y constatar que estas no nos dicen exactamente lo que ocurrió, sino que tenemos que preguntarles e inferir a partir de ellas cómo era la vida en el pasado. Sólo así es posible superar muchas ideas erróneas del alumnado, como la de que el pasado está ya registrado y escrito en los libros y que lo único que cabe hacer es aprendérselo, o que los profesores son quienes mejor se lo saben porque lo han estudiado en los libros de Historia, tal como muestran algunas investigaciones (Shemilt, 1987). Pero además es necesario hacer una aproximación detenida a los hechos del pasado si queremos que estos adquieran sentido para los alumnos. Sólo así lograremos interesarles por el pasado en sí y por el conocimiento histórico, que nos facilita una mejor comprensión de nuestro presente y futuro. Al final la historia es conocimiento y reflexión sobre la experiencia humana y no solo, ni mucho menos, una sucesión de hechos que se memorizan para olvidar a los pocos días (aunque, afortunadamente, podamos recuperarlos en segundos con la tecnología actual). En suma, considero que los contenidos sustantivos son imprescindibles, pues no se puede trabajar sobre la nada, pero los contenidos metahistóricos, de carácter más estratégico son también fundamentales en la medida en que son, precisamente, los que le otorgan sentido y razón de ser a la Historia en la escuela.

MJMR: En relación a la evaluación, cuya importancia en el proceso de enseñanza y aprendizaje podríamos considerar que radica en su naturaleza como procedimiento determinante para el diseño de las fases anteriores en el planteamiento didáctico, ¿qué aspectos reconoce como esenciales a la hora de concretar una evaluación que atienda al desarrollo de la competencia histórica?

JDC: Cuando era pequeño, en la clase de caligrafía nos daban unos pequeños rótulos que debíamos copiar a lo largo de toda una página. Algunos aludían a refranes populares que eran una forma de educarnos. Recuerdo especialmente uno que decía: "Dime con quién andas y te diré quién eres". Pues bien, en un artículo (Domínguez, 1994) de hace ya años parafraseé ese refrán escribiendo "dime cómo evalúas y te diré qué enseñas". Y es que, en más de una ocasión he considerado que la primera tarea a la hora de elaborar una unidad didáctica es diseñar la evaluación, porque al hacerlo, estoy plasmando exactamente qué es lo que quiero que aprendan mis estudiantes de aquí a unas semanas. La evaluación debe estar en el eje de la enseñanza, porque plantearse qué es lo que queremos que nuestros alumnos y alumnas aprendan, equivale a concretar con precisión, y no en objetivos demasiado vagos, a dónde queremos llegar.

Afortunadamente, en los últimos años trabajé en el Instituto de Evaluación del Ministerio de Educación, donde tuve la ocasión de estar en contacto con estudios de evaluación internacionales 
como PISA, PIRLS y otros. Esos estudios tienen un gran valor didáctico para mí, pues evalúan competencias, es decir, la capacidad del estudiante para resolver un problema o una situación poniendo en juego los conocimientos que ha adquirido en la escuela.

Al respecto hay un ejercicio de PISA que siempre pongo de ejemplo porque resulta muy ilustrador. El estímulo de la pregunta incluye una imagen con seis dados y el enunciado señala que "el número total de puntos de las dos caras opuestas de cada dado es siempre siete". A partir de ello se pregunta: ¿qué número de puntos tendrá la cara inferior de cada dado?”. Llama la atención que la prueba solo requiera al alumno hacer una simple resta (siete, menos el número que muestre cada dado en su cara superior), procedimiento que estoy seguro pueden realizar correctamente todos los jóvenes de 16 años; sin embargo, en ese ejercicio hubo un $32 \%$ de errores de media en los países de la OCDE, es decir, casi uno de cada tres alumnos de 15 años no supo responder a la pregunta, seguramente no porque no supiera restar, sino porque no comprendió bien lo que se le estaba preguntando. Es un ejemplo para ilustrar que la prueba de evaluación tiene que plantear cuestiones que exijan aplicar el conocimiento, (la resta en este caso), a una situación concreta. Y encontrar esa forma de aplicar el conocimiento a un problema específico es el quid de la cuestión.

La prueba PISA en ciencias experimentales evalúa tres grandes competencias: "Explicar científicamente los fenómenos", "Identificar y comprender cuestiones científicas" e "Interpretar las pruebas científicas". Pues bien, cada una de las preguntas o ítems de la prueba tiene como fin evaluar una de esas competencias. Así, por ejemplo, para evaluar la segunda de esas competencias se utilizó esta pregunta: En clase, los alumnos han hecho un experimento para comprobar que la lluvia ácida corroe el mármol de las estatuas. Para ello, han sumergido una astilla de mármol en vinagre (similar a la lluvia ácida) y otra igual en agua destilada. ¿Puedes explicar por qué han introducido el mármol en agua destilada? Es decir, la pregunta evalúa si el alumno conoce el papel clave que tiene el control de variables en un experimento científico.

Siguiendo este modelo de PISA para las ciencias experimentales, he planteado la evaluación de la historia a partir de tres grandes competencias. La primera, "Explicar históricamente hechos del pasado o del presente", evalúa la aplicación de los conocimientos históricos (contenidos) de la época (cronología, contexto político, económico, social, cultural, etc.) a hechos concretos no estudiados previamente. La segunda, "Utilizar las pruebas históricas", evalúa el trabajo con las fuentes. Por último, la competencia "Comprender la lógica del conocimiento histórico", trata de evaluar aspectos como la explicación causal, por empatía y los procesos de cambio y continuidad a lo largo del tiempo.

Estas grandes competencias recogen según muchos autores, los conceptos básicos del pensamiento histórico por lo que también deben ser los aspectos esenciales a la hora de concretar la evaluación de la educación histórica. Y llegados a este punto, cabe subrayar que esto no significa que podamos olvidarnos del conocimiento de tipo conceptual, los contenidos, al contrario, hace falta tener un mínimo marco de la evolución histórica y del contexto de la época, para poner en juego adecuadamente esas destrezas de pensamiento.

MJMR: Actualmente estamos llevando a cabo una investigación sobre el desarrollo del pensamiento histórico a través del trabajo con fuentes materiales, concretamente estamos muy interesados en las fuentes objetuales. ¿Qué beneficios educativos identifica que puede tener para el alumnado el trabajo con evidencias históricas $y$, en concreto, con fuentes objetuales, y qué recomendaciones metodológicas sugiere al respecto?

JDC: Recuerdo que Joan Santacana (2012) nos enseñaba hace un par de años unas diapositivas en las que aparecían las gafas rotas de Salvador Allende descubiertas unos días después del golpe de Estado de Pinochet en 1973. Una mujer las encontró y las guardó en su bolsillo y no fue hasta 1996, acabada la dictadura, cuando finalmente las donó al Museo Histórico Nacional de Chile, ya que en ausencia de democracia, tenía miedo de sufrir algún tipo de represalia. Sin duda es un objeto muy elocuente y muy importante en la historia de Chile. A mí personalmente 
me produjo una emoción tremenda ver esa imagen, porque evocó en mí los recuerdos de aquel golpe de estado tan salvaje en Chile. Aunque sea un objeto sin valor material, considerando las sensaciones que produjo en mí, que soy una persona que vive a miles de kilómetros de Chile, imagino lo que pueden significar esas gafas rotas para muchos chilenos. Esa emoción que provocan los objetos, que nos abren de pronto una puerta a un mundo alejado, tapado totalmente por el paso del tiempo, es la gran virtud que tienen las fuentes materiales. $Y$ ha de ser aprovechado por los docentes para despertar emoción en el alumnado, algo que es sin duda clave, para motivarles por el conocimiento, por aprender y saber. Por supuesto, además de sentimientos, el contacto directo con restos materiales, facilita hacerle pensar y reflexionar al estudiante, conectando con ese objeto sus conocimientos. Las fuentes materiales, al permitirnos vincular pensamientos y sentimientos, son una poderosa garantía de aprendizaje.

MJMR: ¿Podría mencionarnos cuáles son a su parecer las medidas o vías de acción necesarias en nuestro sistema educativo para que la Historia sea trabajada finalmente y de forma generalizada como una enseñanza que fomente el pensar históricamente?

JDC: Veamos qué fue lo que se probó y no funcionó con la LOGSE de 1990. Por un lado, se legisló desde arriba un nuevo currículo que debía caer en cascada desde el Ministerio a los centros educativos. Por otro lado, se pusieron en marcha cursos de formación para los profesores en servicio. Además, el Ministerio impulsó la elaboración, de materiales para el trabajo en las aulas. Sin embargo, nada de ello parece haber funcionado como inicialmente se pensó. Es cierto que la aplicación posterior de la LOGSE por los gobiernos populares la desvirtuó en buena medida, pero lo es también que la ESO no ha sabido hasta hoy encauzar de forma apropiada la diversidad de intereses y conocimientos del alumnado. Y eso ha sido, en mi opinión, una de las principales causas de desafección de muchos docentes por su profesión.

Actualmente PISA y otras evaluaciones internacionales, además de influir notoriamente en las políticas educativas de muchos países, inciden en la práctica de los profesores, muchos de los cuales nos planteamos la importancia que tiene saber utilizar el conocimiento en la vida real. Por aportar un ejemplo, muchos profesores de lengua dedican ahora más tiempo y atención a que los alumnos aprendan a leer comprensivamente y menos a que aprendan gramática. Creo que las pruebas externas pueden ser otro instrumento más para mejorar la enseñanza, además del currículo y los materiales didácticos. Por mi experiencia docente en Secundaria, conozco de primera mano cómo la prueba de Selectividad pudo llegar a influir en mi trabajo, pues me instaba a ejercitar a mis alumnos para que cuando miraran una obra de arte, por ejemplo, supieran analizar, desde los aspectos técnicos y estéticos de la obra hasta su interpretación en el contexto histórico. Si en lugar de preguntar por conocimientos memorizados se elaboran pruebas donde debas poner en juego tus conocimientos para manejar e interpretar información nueva para ti, resolver pequeños problemas y reflexionar sobre cuestiones de hoy y del futuro, entonces creo que una prueba de evaluación puede ser realmente útil.

Pero además de las evaluaciones, el currículo y los materiales didácticos, no cabe olvidar la formación del profesorado, como vías de acción para enseñar a pensar históricamente. Sin una adecuada formación y protagonismo del profesorado, nada va a funcionar.

MJMR: Para terminar, nos gustaría referirnos al libro que recientemente ha publicado, Pensamiento histórico y evaluación de competencias (Domínguez, 2015). ¿Nos podría comentar cuáles son sus principales aportaciones y a qué público va dirigido?

JDC: Es un libro que recoge en primer lugar lo que he estudiado y aprendido de mis maestros, en su mayoría anglosajones, pero también españoles. A partir de sus investigaciones, experiencias y reflexiones sobre el pensamiento histórico, he intentado dar el paso siguiente para concretar ese pensamiento histórico en destrezas que permitan evaluarlo. $Y$ es que el libro presta especial 
atención a traducir en la práctica la reflexión teórica. Para ello se ha elaborado un ejemplo de prueba de evaluación, para $4 .^{\circ}$ de ESO, que aunque no ha podido incluirse como Anexo en el libro, puede descargarse libremente de la web de la editorial Graó ${ }^{2}$. Traducir el pensamiento histórico en destrezas evaluables y acompañar todo ello de un ejemplo de prueba que lo traduzca en la práctica, son en mi opinión las principales aportaciones del libro. Para ello me ha sido muy instructivo el contacto directo con las pruebas de PISA y PIRLS, que muestran cómo las grandes competencias educativas, es necesario traducirlas en destrezas cognitivas que puedan ser evaluables. Así, para evaluar el trabajo con las fuentes, se necesita definir destrezas de comprensión lectora y también destrezas historiográficas. Hace falta, primero, comprobar que el alumnado comprende lo que lee, y eso se traduce entre otras cosas, en las destrezas de obtención der información explícita e implícita en el texto. Pero además de la comprensión lectora, se requieren otras destrezas que son específicas del historiador como, por ejemplo, saber contextualizar las fuentes en su época, analizarlas en detalle y de manera crítica, cotejarlas, etc. Por ejemplo, en una de las preguntas de nuestra prueba, se pide al estudiante que explique a un compañero que acaba de llegar a España una frase de un texto que describe

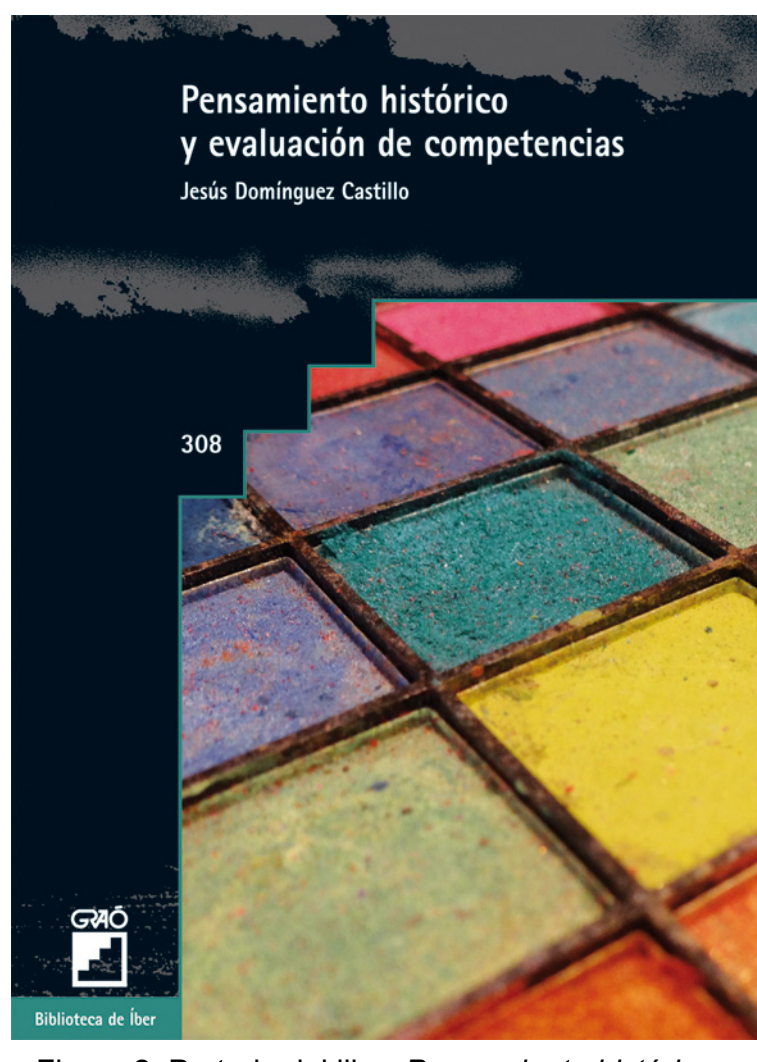

Figura 2: Portada del libro Pensamiento histórico y evaluación de competencias. Publicado en Barcelona por la Editorial Graó.

(Fuente: www.grao.com).

la vida de una familia de agricultores humildes en la España de 1950. La frase dice en concreto que los ricos propietarios del pueblo no daban trabajo como jornalero al marido, por estar casado con la hija de un "rojo" muerto en la "guerra". Esta pregunta, además de comprensión lectora, exige un mínimo conocimiento del contexto social y político de la posguerra española. En definitiva, dentro de cada una de las grandes competencias históricas hay una serie de destrezas de pensamiento que se traducen en preguntas concretas de evaluación.

El libro va dirigido principalmente a los alumnos de los cursos de Máster de Formación del Profesorado, porque puede ser una herramienta muy útil para el estudio, a la vez teórico y práctico, de lo que significa pensar históricamente y cómo plantear su evaluación. Por ello el libro va acompañado de una prueba de evaluación que, además de alternativa a la tradicional prueba conceptual y memorística, puede ser también utilizada como material de enseñanza y como base para promover el debate y la reflexión. Ese es el objeto del libro y espero que pueda ser útil, especialmente a los actuales y futuros docentes de Secundaria.

\section{Referencias}

Domínguez, J. (1989). El lugar de la historia en el currículum 11-16. Un marco general de referencia. En M. Carretero, J. I. Pozo y M. Asensio (Comps.), La enseñanza de las Ciencias Sociales (pp. 33-60). Madrid: Visor.

1 La prueba la forman dos unidades de evaluación: Emigrantes Españoles de ayer y de hoy y El trabajo infantil en la Revolución industrial y en la actualidad, accesibles en:

http://www.grao.com/dades/BB308/BB308ANEXO_U1.pdf

http://www.grao.com/dades/BB308/BB308ANEXO_U2.pdf 
Domínguez, J. (1994). Una propuesta de categorías y niveles para la evaluación del aprendizaje en el área de ciencias sociales, geografía e historia. En Grupo Ínsula Barataria (Coord.), Enseñar y aprender Ciencias Sociales. Algunas propuestas de Modelos Didácticos (pp. 229252). Madrid: Mare Nostrum.

Domínguez, J. (2015). Pensamiento histórico y evaluación de competencias. Barcelona: Graó.

Ley Orgánica 1/1990, de 3 de octubre, de Ordenación General del Sistema Educativo (LOGSE). Boletín Oficial del Estado (BOE), 238, de 4 de octubre de 1990, 28927-28942.

Ley Orgánica 8/2013, de 9 de diciembre, para la Mejora de la Calidad Educativa (LOMCE). Boletín Oficial del Estado (BOE), 295, de 10 de diciembre de 2013, 97858-97921.

López Facal, R. (2003). La enseñanza de la historia, más allá del nacionalismo. En J. J. Carreras Ares y C. Forcadell Álvarez (Eds.), Usos Públicos de la Historia. Ponencia en el VI Congreso de la Asociación de Historia Contemporánea (pp. 19-21). Madrid: Marcial Pons y Prensas Universitarias de Zaragoza.

Santacana, J. (2012). Museos de historia, cadáveres elegantes del pasado. Recuperado de www.didcticadelpatrimonicultural.blogspot.com.es/2012/04/museos-de-historia-cadavereselegantes.html

Shemilt, D. (1987). Adolescents ideas about evidence and methodology in history. En C. Portal (Ed.), The History Curriculum for Teachers (pp. 39-61). London: The Falmer Press. 



\section{Panta Rei}

PANTA REI es una revista digital de investigación orientada a la Historia y otras ciencias afines. Su principal objetivo es la transmisión del conocimiento científico, dando una oportunidad también a los jóvenes investigadores que quieren abrirse camino en el estudio de las ciencias humanas y sociales. Se compone de estudios originales relacionados con la disciplina histórica así como su didáctica y difusión. Las diferentes secciones que componen la revista son: artículos de investigación, entrevistas a profesionales, recensiones de monografías de actualidad y crónicas de congresos o eventos científicos relevantes.

Todos los artículos publicados son objeto de un proceso de revisión a cargo de un mínimo de dos evaluadores, que se consideran expertos en el ámbito temático del artículo propuesto. Nuestro deseo es poder ofrecer unos contenidos rigurosos, de calidad y de interés.

EI CEPOAT (Centro de Estudios del Próximo Oriente y la Antigüedad Tardía de la Universidad de Murcia) es la institución encargada de la coordinación y gestión de la revista, desde donde anualmente se lanzará la convocatoria para aquellos que estén interesados en publicar sus trabajos, siempre relacionados con la Historia, Arqueología, Historia del Arte, Didáctica de las Ciencias Sociales, etc.

PANTA REI is a digital journal focused on History and other sciences related to it. Its main objective is the transmission of scientific knowledge by giving also an opportunity to young researchers who want to make their way in the study of human and social sciences. It is composed by original studies related to History, as well as its didactics and promotion. The different sections of this journal are: research articles, interviews to professionals, recensions on monographs about current issues and reports about congresses or relevant scientific events.

All the articles published are subject to a revision process carried out by a minimum of two reviewers who are considered to be experts in the field of the article proposed. Our wish is to offer rigorous contents with quality and being of interest to the reader.

CEPOAT (Centre of Studies of the Middle East and Late Antiquity of the University of Murcia) is the institution in charge of the coordination and management of this journal. This is the centre from where the call for papers will be launched for all the people interested in publishing their papers, always related to History, Archeology, Art History, Didactics of the Social Sciences, etc. 


\section{Normas de Publicación}

El autor se compromete a enviar trabajos originales, que no se encuentren publicados en otras revistas ni en otros idiomas. Así mismo, el mismo artículo no podrá ser presentado en otras revistas mientras dure el proceso de evaluación.

\section{Envío y presentación de originales}

Los artículos se enviarán exclusivamente a través del correo electrónico a la dirección pantarei@um.es. Los textos serán enviados en formato DOC y las imágenes en formato JPEG o TIFF, y con un tamaño mínimo de 2000 px. Éstas no aparecerán incorporadas en el texto, sino enviadas en archivo aparte y correctamente numeradas según su posición en el texto. Junto al trabajo, se rellenará y enviará un documento aparte en el que se especifiquen los datos del autor siguiendo el modelo disponible en la página Web de la revista.

Para la redacción de los trabajos se tendrá en cuenta el Manual de la American Psychological Association, en su sexta edición. La extensión máxima de los trabajos será de 30 páginas. La tipografía será Arial 11, con interlineado sencillo y sin espacio alguno entre párrafos. El texto deberá ir justificado a ambos márgenes y sin sangría en los primeros párrafos. Los márgenes serán de $2,50 \mathrm{~cm}$. En los casos en los que fuera necesario incorporar notas, éstas irán a pie de página, enumeradas consecutivamente, con tipografía Arial 10, interlineado sencillo y justificadas a ambos márgenes.

Una información más detallada se encuentra disponible en la página http://www.um.es/cepoat/ pantarei.

\section{Proceso de valoración y evaluación}

Una vez recibidos los trabajos, la Revista realizará una primera valoración. Si el trabajo enviado se ajusta a las normas de presentación propuestas, la temática es coincidente con la línea editorial de la revista y posee la calidad científica necesaria, será remitido al consejo asesor para una primera evaluación. Si no es así en este primer paso se puede rechazar directamente los documentos que incumplan claramente la línea editorial.

Será el Consejo Asesor quien indique a la revista la originalidad, relevancia, estructura, redacción, aparato bibliográfico, etc. del trabajo enviado y, para ello, se designará a dos revisores expertos externos que evaluarán cada uno de los trabajos, que pueden formar parte (o no) de este Consejo Asesor. La selección de los revisores se ajustará a la temática y características metodológicas del trabajo. El nombre y filiación de los autores serán eliminados del trabajo para su revisión, así como los revisores actuarán de manera anónima y confidencial.

Los revisores deberán rellenar un informe de evaluación que centrará su atención en aspectos tales como características formales, originalidad y novedad de los trabajos, relevancia de las propuestas y los resultados, calidad metodológica y validez científica.

Una vez terminado el proceso se decidirá la aceptación o no de los mismos y su publicación en el número que sea pertinente, así como las modificaciones susceptibles de ser realizadas para su final publicación. Dicha notificación se enviará únicamente por correo electrónico, en un plazo máximo de seis meses. 


\section{Publishing rules}

The author is committed to submit original papers not having been published in other reviews or in other languages. In this way, it is not allowed for the same paper to be presented in other reviews during the evaluation process.

\section{Submission and presentation of originals}

The articles will be exclusively submitted by email to pantarei@um.es. The texts will be submitted in DOC format and the images in JPEG or TIFF format, and with a minimum size of 2000 px. Images will not be integrated in the text but sent in another file and properly numbered according to their position in the text. Attached to the paper, a document will be filled out and sent where the author's data will be specified following the model available on the website.

The sixth edition of the Manual of the American Psychological Association will be taken into account for the writing of the papers. The length of the papers must not exceed 30 pages. Typography will be Arial 11 , with simple line spacing and no space between paragraphs. The text must be justified on both margins without indentation in the first paragraphs. Margins size will be $2.50 \mathrm{~cm}$. Where it could be necessary the incorporation of notes, they will be at the bottom of the page, consecutively numbered with typography Arial 10, simple line spacing and justified on both margins.

More detailed information is available on the website: http://www.um.es/cepoat/pantarei.

\section{Examination and assessment process}

The Journal will submit the papers to a first examination once received. If the paper follows the presentation guidelines, the subject agrees with the editorial line of this journal, and possess the scientific quality required, it will be sent to the advisory council for a first assessment. If not, the documents which clearly fail to complete the editorial line may be rejected straightaway in this first step.

The Advisory Council will indicate the originality, relevance, structure, writing, bibliography, etc. of the text to the journal; for this purpose, two outside experts will be designated to review the papers; these experts can be (or not) part of this Advisory Council. The selection of the experts will adjust to the subject and methodological characteristics of the paper. Name and affiliation of the author will be eliminated from the text for its review, in this way experts will act anonymously and confidentially.

The experts will fill out an assessment report which will focus on aspects such as formal characteristics, originality and novelty of the papers, relevance and results of the proposal, methodological quality and scientific validity.

Once the process is finished, the acceptance or not of the papers and its publication in the corresponding edition will be decided, as well as the modifications that may be done for its final publication. This notification will be sent by email within 6 months maximum. 

